\title{
Incident-energy and system-size dependence of directed flow
}

\author{
Gang Wang (for the STAR $\ddagger$ Collaboration) \\ University of California, Los Angeles, California 90095, USA \\ E-mail: gwang@physics.ucla.edu
}

\begin{abstract}
We present STAR's measurements of directed flow for charged hadrons in $\mathrm{Au}+\mathrm{Au}$ and $\mathrm{Cu}+\mathrm{Cu}$ collisions at $\sqrt{s_{\mathrm{NN}}}=200 \mathrm{GeV}$ and $62.4 \mathrm{GeV}$, as a function of pseudorapidity, transverse momentum and centrality. We find that directed flow depends on the incident energy, but not on the system size. We extend the validity of limiting fragmentation hypothesis to different collision systems.
\end{abstract}

\section{Motivation}

Directed flow is quantified by the first harmonic $\left(v_{1}\right)$ in the Fourier expansion of the azimuthal distribution of produced particles with respect to the reaction plane [1]. It describes the collective sideward motion of produced particles and nuclear fragments, and carries information from the very early stage of the collision [2]. Charged-hadron $v_{1}$ results at RHIC have been previously reported over a wide range of incident energies in $\mathrm{Au}+\mathrm{Au}$ collisions [3, 4, 5, 6], and they support the limiting fragmentation hypothesis [4, 5, 7]. In RHIC run V (2005), lighter nuclei were collided at $\sqrt{s_{\mathrm{NN}}}=200$ $\mathrm{GeV}$ and $62.4 \mathrm{GeV}$, which enables us to study the system-size dependence of directed flow, as well as the incident-energy dependence.

\section{Data sets and the study}

This study is based on eight million $200 \mathrm{GeV} \mathrm{Au+Au}$ events, five million $62.4 \mathrm{GeV}$ $\mathrm{Au}+\mathrm{Au}$ events, twelve million $200 \mathrm{GeV} \mathrm{Cu}+\mathrm{Cu}$ events, and eight million $62.4 \mathrm{GeV}$ $\mathrm{Cu}+\mathrm{Cu}$ events. All of them were obtained from a minimum-bias trigger. All errors are statistical. Tracks of charged particles are reconstructed by STAR's main TPC [8] and forward TPCs [9], with the pseudorapidity $(\eta)$ coverage of $|\eta|<1.3$ and $2.5<|\eta|<4.0$, respectively. The centrality definition and cuts used are the same as in Ref. [10].

At RHIC energies, it is a challenge to measure $v_{1}$ accurately due to the small signal of $v_{1}$ itself and the large systematic error arising from non-flow correlations. In this talk, STAR's preliminary $v_{1}\{$ ZDC-SMD $\}$ results are obtained with non-flow effects $\ddagger$ For the full list of STAR authors and acknowledgements, see appendix 'Collaborations' in this volume. 
Table 1. The resolution of the 1st-order full event plane provided by STAR ZDCSMDs, as determined from the sub-event correlation between east and west SMDs. The errors in the table are statistical.

\begin{tabular}{c|c|c|c|c}
\hline Centrality & $200 \mathrm{GeV} \mathrm{Au}+\mathrm{Au}$ & $62.4 \mathrm{GeV} \mathrm{Au}+\mathrm{Au}$ & $200 \mathrm{GeV} \mathrm{Cu}+\mathrm{Cu}$ & $62.4 \mathrm{GeV} \mathrm{Cu}+\mathrm{Cu}$ \\
\hline $70 \%-80 \%$ & $0.296 \pm 0.003$ & $0.179 \pm 0.005$ & & \\
\hline $60 \%-70 \%$ & $0.348 \pm 0.003$ & $0.185 \pm 0.004$ & & \\
\hline $50 \%-60 \%$ & $0.382 \pm 0.002$ & $0.176 \pm 0.005$ & $0.140 \pm 0.003$ & $0.039 \pm 0.011$ \\
\hline $40 \%-50 \%$ & $0.397 \pm 0.002$ & $0.167 \pm 0.005$ & $0.144 \pm 0.003$ & $0.043 \pm 0.011$ \\
\hline $30 \%-40 \%$ & $0.390 \pm 0.002$ & $0.138 \pm 0.006$ & $0.147 \pm 0.003$ & $0.032 \pm 0.015$ \\
\hline $20 \%-30 \%$ & $0.365 \pm 0.002$ & $0.110 \pm 0.008$ & $0.121 \pm 0.004$ & \\
\hline $10 \%-20 \%$ & $0.309 \pm 0.003$ & $0.081 \pm 0.010$ & $0.095 \pm 0.005$ & \\
\hline $5 \%-10 \%$ & $0.220 \pm 0.006$ & & $0.059 \pm 0.008$ & \\
\hline $0-5 \%$ & $0.120 \pm 0.002$ & & $0.059 \pm 0.008$ & \\
\hline
\end{tabular}

minimized by determining the event plane from spectator neutrons, the same approach as used in Ref. [5, 6]. The resolution, as defined in Ref. [1], of the first-order event plane reconstructed with STAR ZDC-SMDs [11] is listed in Table 1], The magnitude of the event plane resolution increases with the spectator $v_{1}$ and the detector efficiency of the ZDC-SMDs, and the latter is smaller for a lower incident energy and/or a smaller collision system. The method $v_{1}\{Z D C-S M D\}$ fails in some centralities, where the event plane resolution is consistent with zero.

\section{Results}

Figures in this talk follow the same convention as in Ref. [5].

Fig. 1 shows $v_{1}$ as a function of pseudorapidity, $\eta$, in $30 \%-60 \%$ most central $\mathrm{Au}+\mathrm{Au}$ and $\mathrm{Cu}+\mathrm{Cu}$ collisions at $\sqrt{s_{\mathrm{NN}}}=200 \mathrm{GeV}$ and $64.2 \mathrm{GeV}$. Data points in Fig. 1 fall into two bands, and the higher band corresponds to the lower energy, which reveals the incident-energy dependence of $v_{1}(\eta)$. For the pseudorapidity and centrality range we studied, $v_{1}(\eta)$ in $\mathrm{Au}+\mathrm{Au}$ collisions and $\mathrm{Cu}+\mathrm{Cu}$ collisions are consistent within errors if both of them were obtained at the same incident energy. It is interesting that $v_{1}$ does not change from $\mathrm{Au}+\mathrm{Au}$ collisisons to $\mathrm{Cu}+\mathrm{Cu}$ collisions, while the system size is reduced by $1 / 3$. A good consistency of $v_{1}$ between $\mathrm{Au}+\mathrm{Au}$ and $\mathrm{Cu}+\mathrm{Cu}$ collisions is observed even for the region near midrapidity, where $v_{2}$ in $\mathrm{Cu}+\mathrm{Cu}$ collisions is considerably lower than that in $\mathrm{Au}+\mathrm{Au}$ collisions [12]. Unlike $v_{2} / \epsilon$ which scales with $\frac{1}{S} \frac{d N_{c h}}{d y}$ (interpreted to be the participant density [12] or the system length [13]), $v_{1}$ is found to be independent of the system size. Instead, it scales with the incident energy. A possible explanation to the different scalings for $v_{2} / \epsilon$ and $v_{1}$ might come from the way in which they are developed : to produce $v_{2}$, intensive momentum exchanges among particles are needed (and the number of momentum exchanges is related to the participant density or the system length), while to produce $v_{1}$, one in principle needs only different rapidity losses (related to the incident energy) for particles with different distances to the center of the 


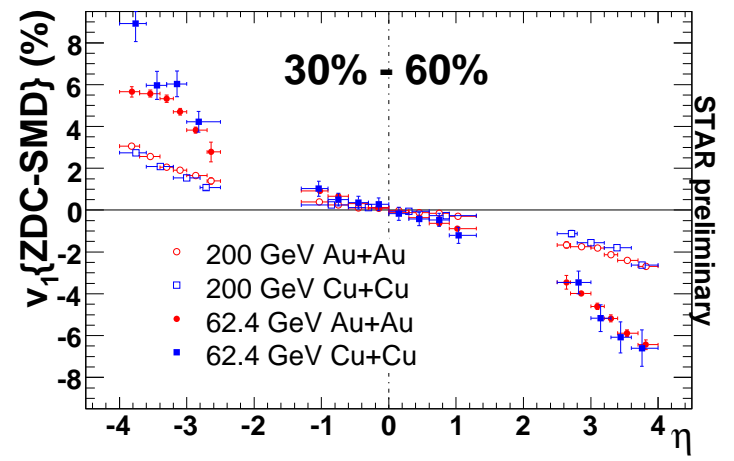

Figure 1. Charged-hadron $v_{1}$ vs. $\eta$, in $\mathrm{Au}+\mathrm{Au}$ Figure 2. Charged-hadron $v_{1}$ vs. $\eta-y_{\text {beam }}$, in and $\mathrm{Cu}+\mathrm{Cu}$ collisions at $200 \mathrm{GeV}$ and $62.4 \mathrm{GeV}$. $\mathrm{Au}+\mathrm{Au}$ and $\mathrm{Cu}+\mathrm{Cu}$ collisions at $200 \mathrm{GeV}$ and 62.4 The plotted errors are statistical.

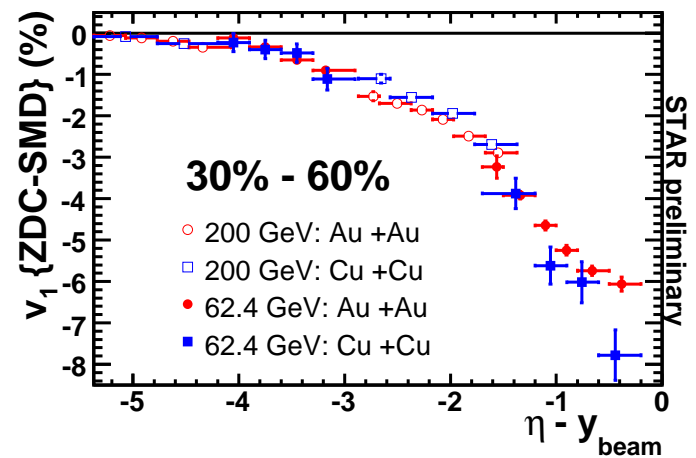

$\mathrm{GeV}$. The plotted errors are statistical.

participant zone.

In Fig. 2, we re-arrange the data points of Fig. 1 in the projectile frame, where zero on the horizontal axis corresponds to the beam rapidity, $y_{\text {beam }}$, for each of the incident energies. Within three units from $y_{\text {beam }}$, most data points fall into a universal curve of $v_{1}$ as a function of $\eta-y_{\text {beam }}$. This incident-energy scaling behavior of directed flow suggests that the limiting fragmentation hypothesis [7] holds even for different collision systems.

The transverse-momentum $\left(p_{t}\right)$ dependence of $v_{1}$ for charged hadrons is shown in Fig. 3. The upper panel shows the results measured in the main TPC, and the lower panel, in the forward TPCs. Since $v_{1}\left(\eta, p_{t}\right)$ is asymmetric about $\eta=0$, the integral of $v_{1}\left(\eta, p_{t}\right)$ over a symmetric $\eta$ range goes to zero. We change $v_{1}\left(\eta, p_{t}\right)$ of particles with negative $\eta$ into $-v_{1}\left(-\eta, p_{t}\right)$, and integrate over all $\eta$. At $200 \mathrm{GeV}, v_{1}\left(p_{t}\right)$ in $\mathrm{Au}+\mathrm{Au}$ and $\mathrm{Cu}+\mathrm{Cu}$ collisions are very close to each other (better visible in the forward pseudorapidity region), and $v_{1}\left(p_{t}\right)$ saturates above $p_{t} \approx 0.8 \mathrm{GeV} / c$. At $62.4 \mathrm{GeV}, v_{1}\left(p_{t}\right)$ reaches its maximum in magnitude at $p_{t} \approx 0.6 \mathrm{GeV} / c$, then saturates $(|\eta|<1.3)$ or slightly decreases $(2.5<|\eta|<4.0)$. Within statistical errors, the incident-energy scaling holds in $v_{1}\left(p_{t}\right)$ as well.

Fig. 4 shows charged-hadron $p_{t}$-integrated $v_{1}$ as a function of centrality for the two collision systems at $200 \mathrm{GeV}$ and $62.4 \mathrm{GeV}$, and the results are presented in two panels defined in the same way as Fig. 3, On the whole, the magnitude of integrated $v_{1}$ approaches zero in central collisions and increases as the collisions become more peripheral. At $200 \mathrm{GeV}$, the $p_{t}$-integrated $v_{1}$ in $\mathrm{Au}+\mathrm{Au}$ collisions is consistent with that in $\mathrm{Cu}+\mathrm{Cu}$ collisions in almost all centrality bins, in regions both near the midrapidity $(|\eta|<1.3)$ and away from it $(2.5<|\eta|<4.0)$. This system-size independence is seemingly true at $62.4 \mathrm{GeV}$. We can see that the incident-energy scaling of $v_{1}$ works well as a funtion of centrality, instead of the participant density, with which the $v_{2} / \epsilon$ scales [12]. This indicates that the initial geometrical shape, but not the density of produced particles, plays an important role in the formation of directed flow. 

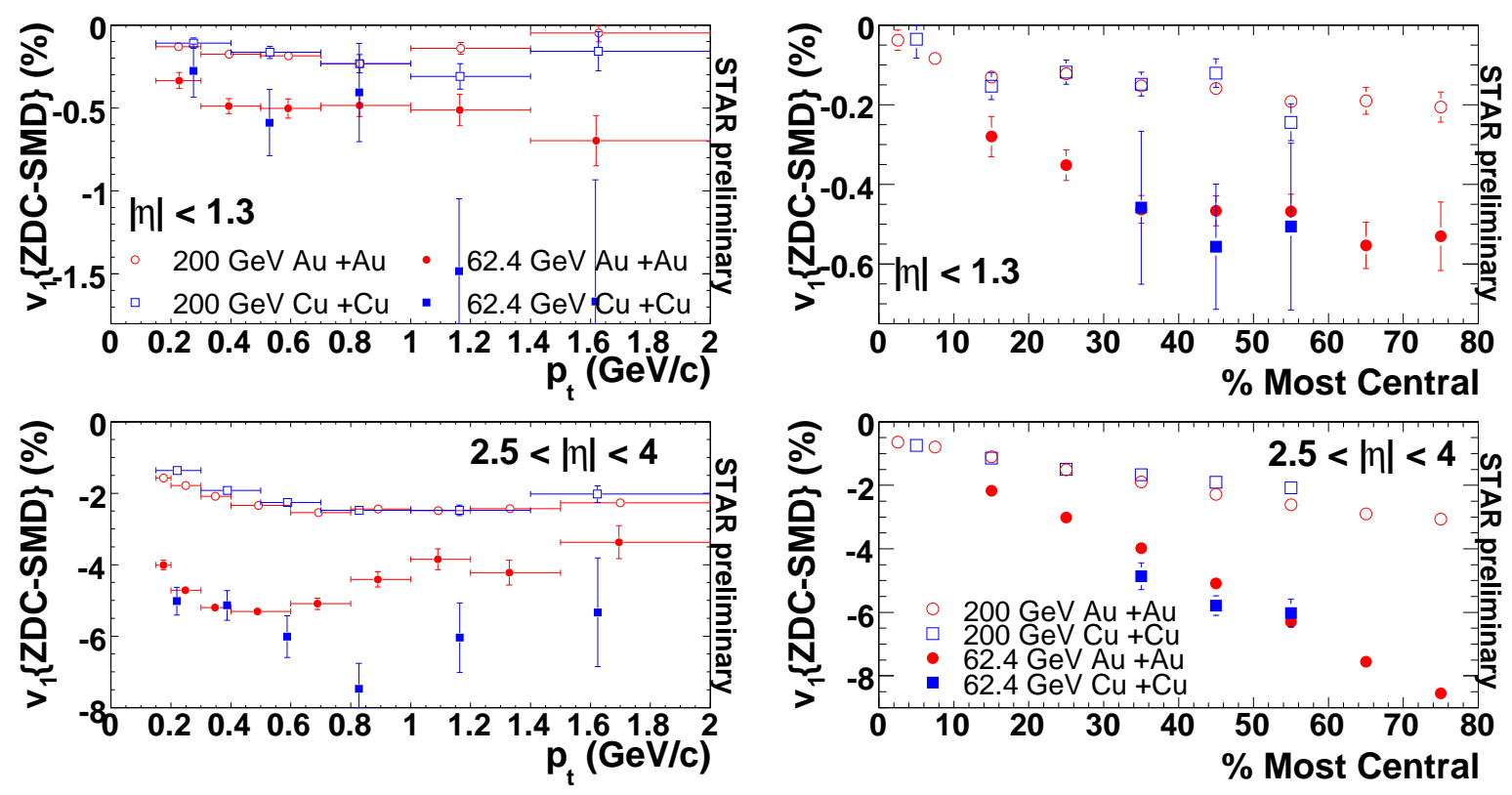

Figure 3. Charged-hadron $v_{1}$ vs. $p_{t}$, in $30 \%-60 \%$ $\mathrm{Au}+\mathrm{Au}$ and $\mathrm{Cu}+\mathrm{Cu}$ collisions at $200 \mathrm{GeV}$ and 62.4 $\mathrm{GeV}$. The upper panel shows the results measured in the main TPC, and the lower panel, in the forward TPCs. The plotted errors are statistical.

Figure 4. Charged-hadron $p_{t}$-integrated $v_{1}$ vs. centrality, in $\mathrm{Au}+\mathrm{Au}$ and $\mathrm{Cu}+\mathrm{Cu}$ collisions at 200 $\mathrm{GeV}$ and $62.4 \mathrm{GeV}$. The upper panel shows the results measured in the main TPC, and the lower panel, in the forward TPCs. The plotted errors are statistical.

\section{References}

[1] Poskanzer A M and Voloshin S A 1998 Phys. Rev. C 581671

[2] Adams J et al (STAR Collaboration) 2005 Nucl. Phys. A 757 102, and refs. therein

[3] Adams J et al (STAR Collaboration) 2004 Phys. Rev. Lett. 92062301

[4] Back B B et al (PHOBOS Collaboration) 2004 J. Phys. G 30 S1243; Back B B et al (PHOBOS Collaboration) 2006 Phys. Rev. Lett. 97012301

[5] Adams J et al (STAR Collaboration) 2006 Phys. Rev. C 73034903

[6] Wang G et al (STAR Collaboration) 2006 Nucl. Phys. A 774515

[7] Benecke J, Chou T T, Yang C -N and Yen E 1969 Phys. Rev. 1882159

[8] Anderson M et al 2003 Nucl. Instr. Meth. A 499659

[9] Ackermann K H et al 2003 Nucl. Instr. Meth. A 499713

[10] Adams J et al (STAR Collaboration) 2005 Phys. Rev. C 72014904

[11] Adler C et al 2001 Nucl. Instr. Meth. A 470 488; The STAR ZDC-SMD has the same structure as the STAR EEMC SMD: Allgower C E et al 2003 Nucl. Instr. Meth. A 499 740; Crawford H et al 2003 STAR ZDC-SMD proposal STAR Note SN-0448

[12] Back B B et al (PHOBOS Collaboration) 2006 Preprint nucl-ex/0610037 Voloshin S A et al (STAR Collaboration) these proceedings

[13] Tang A H these proceedings 